\title{
Internuclear gene silencing in Phytophthora infestans is established through chromatin remodelling
}

Correspondence

Pieter van West

p.vanwest@abdn.ac.uk

Received 3 December 2007

Revised 20 February 2008

Accepted 25 February 2008
Pieter van West, ${ }^{1}$ Samantha J. Shepherd, ${ }^{1}$ Claire A. Walker, ${ }^{1}$ Shuang Li, ${ }^{1}$ Alex A. Appiah, ${ }^{1}$ Laura J. Grenville-Briggs, ${ }^{1}$ Francine Govers ${ }^{2}$ and Neil A. R. Gow ${ }^{1}$

${ }^{1}$ Aberdeen Oomycete Group, College of Life Sciences and Medicine, Institute of Medical Sciences, University of Aberdeen, Foresterhill, Aberdeen AB25 2ZD, UK

${ }^{2}$ Laboratory of Phytopathology, Binnenhaven 5, 6709 PD Wageningen, The Netherlands

In the plant pathogen Phytophthora infestans, nuclear integration of inf1 transgenic DNA sequences results in internuclear gene silencing of inf1. Although silencing is regulated the transcriptional level, it also affects transcription from other nuclei within heterokaryotic cells of the mycelium. Here we report experiments exploring the mechanism of internuclear gene silencing in $P$. infestans. The DNA methylation inhibitor 5-azacytidine induced reversion of the inf1-silenced state. Also, the histone deacetylase inhibitor trichostatin-A was able to reverse inf1 silencing. inf1-expression levels returned to the silenced state when the inhibitors were removed except in non-transgenic inf1-silenced strains that were generated via internuclear gene silencing, where inf1 expression was restored permanently. Therefore, inf1-transgenic sequences are required to maintain the silenced state. Prolonged culture of non-transgenic inf1-silenced strains resulted in gradual reactivation of inf1 gene expression. Nuclease digestion of inf1-silenced and nonsilenced nuclei showed that inf1 sequences in silenced nuclei were less rapidly degraded than non-silenced inf1 sequences. Bisulfite sequencing of the endogenous inf1 locus did not result in detection of any cytosine methylation. Our findings suggest that the inf1-silenced state is based on chromatin remodelling.

\section{INTRODUCTION}

In many organisms, including animals, plants, fungi and protists, transcriptional gene silencing (TGS) can occur as a consequence of the introduction of transgenes into the somatic genome (Selker, 1997; Henikoff, 1998; Vaucheret et al., 1998; Cogoni \& Macino, 1999; van West et al., 1999). TGS is thought to have evolved as a mechanism to protect the nuclear genome against potentially harmful transforming sequences such as transposons and viruses (Kooter et al., 1999). TGS is reminiscent of paramutation found in plants, and other imprinting phenomena (Hollick et al., 1997). Paramutation is an epigenetic phenomenon in which one allele or locus spontaneously acquires a less transcriptionally active state, which is then imposed on homologous sequences in the genome (Chandler et al., 2000). In plants and animals TGS is often, but not invariably associated with altered methylation patterns and

Abbreviations: 5-AC, 5-azacytidine; BuA, butyric acid; HDAC, histone deacetylase; PTGS, post-transcriptional gene silencing; TGS, transcriptional gene silencing; TSA, trichostatin A. changes in chromatin structure. Organisms lacking efficient DNA methylation systems, such as Drosophila, Caenorhabditis elegans and yeast, use chromatin modification to achieve imprinting and to maintain epigenetic states. Several experiments have shown that chromatin remodelling and histone deacetylation processes influence higher-order chromatin structure and can result in transcriptional repression (Tyler \& Kadonaga, 1999; Grewal \& Elgin, 2002; Vermaak et al., 2003).

Other homology-dependent gene-silencing phenomena are also found; these are thought to occur at a posttranscriptional level in the cytoplasm. Post-transcriptional gene silencing (PTGS) phenomena have one major feature in common, which is that target mRNAs are degraded in a sequence-specific manner. Examples include RNA interference (RNAi) (Fire et al., 1998; Plasterk \& Ketting, 2000), co-suppression in plants (van der Krol et al., 1990), quelling in fungi (Cogoni et al., 1996), and virus-induced gene silencing (VIGS) in plants (Ruiz et al., 1998; Lu et al., 2003). It is likely that PTGS phenomena have also evolved as genome defence responses and/or as regulatory mechanisms of gene expression (Baulcombe, 2002). 
We have shown previously that transformation of the diploid oomycete plant pathogen Phytophthora infestans with constructs of the infl elicitin gene resulted in gene silencing of both the transgenes and the endogenous gene (van West et al., 1999). This silencing phenomenon in $P$. infestans, termed internuclear gene silencing, is in some aspects comparable to quelling, found in Neurospora crassa (Cogoni et al., 1996; for a review see Nakayashiki, 2005). As in Neurospora, gene silencing in P. infestans is dominant, and acts in trans in heterokaryotic strains, suggesting the involvement of a cytoplasmic diffusible molecule. However, instead of inducing mRNA degradation (PTGS), the diffusible signal in $P$. infestans apparently acts at the transcriptional level (van West et al., 1999). Another distinction from $N$. crassa quelling is that the silenced state is persistent even in non-transgenic homokaryotic progeny. Here we describe evidence that the TGS phenomenon in $P$. infestans is mediated by changes in histone deacetylation.

\section{METHODS}

P. infestans strains and culture conditions. The wild-type $P$. infestans strain 88069 was used in all experiments. inf1 antisense transformants (PY31 and PY37), promoterless inf1-cDNA transformant (SY21), homokaryotic non-inf1-transgenic but inf1-silenced strains $(\mathrm{H} 1, \mathrm{H} 2, \mathrm{H} 3, \mathrm{H} 4$ and $\mathrm{H} 5)$, and a transgenic control strain (Y15) have the 88069 genetic background and were obtained as described by van West et al. (1999). All transgenic strains were cotransformed with the geneticin resistance gene nptII, except for $\mathrm{H} 1$, $\mathrm{H} 2, \mathrm{H} 3, \mathrm{H} 4$ and $\mathrm{H} 5$ which express the hygromycin resistance gene $h p t$. Cultures were grown routinely in the dark at $18{ }^{\circ} \mathrm{C}$ on ryesucrose (RS) medium as described previously (van West et al., 1998). Mycelium for isolation of DNA and RNA was obtained by growing cultures of $P$. infestans in liquid RS-medium or modified Plich medium (van West et al., 1999). When required, trichostatin A (TSA; Calbiochem), butyric acid (BuA; Sigma) and 5-azacytidine (5-AC; Calbiochem) were added to the medium prior to inoculation. Final concentrations were $3.3 \mu \mathrm{M}$ TSA, $5 \mathrm{mM}$ BuA and $50 \mu \mathrm{M}$ 5-AC.

Detection of potential DNA methylation in genomic DNA. To detect cytosine methylation in the inf1 gene, the EZ DNA Methylation kit (Zymo Research) protocol was followed according to the instructions supplied by the company. Genomic DNA was treated with bisulfite, resulting in the conversion of unmethylated cytosines to uracil. Methylated cytosines will not undergo conversion. To detect the presence of methylated cytosines, the treated genomic DNA of strains 88069, Y15, PY31, PY37 and H1 was amplified by PCR to allow analysis by DNA sequencing. PCR reactions were carried out using primers (inf1Fm, $5^{\prime}$-TTTGTGAAGGTGTTATTTT- $3^{\prime}$; inf1Rm, $5^{\prime}$-CATACAAATACACATTAAATAC-3') that had been modified to account for conversion of the $\mathrm{C}$ into a $\mathrm{U}$, and primers which were not modified (inf1F, 5'-CTTGTGAAGGCGTCATTCC-3'; inf1R, 5' CGTACGAGTACACGTTGAGTAC-3'). PCR products were only obtained in reactions using the modified primers. To improve sensitivity of the PCR reaction and reduce contaminants, a further nested PCR was carried out. Therefore, a second set of modified primers was designed internally to the original primers (inf1Fmn, $5^{\prime}$ TTTGTTGTAGGATTAGATA-3'; inf1Rmn, 5'-CAAACCACTAATTAACACC- $3^{\prime}$ ). Gel purification of the generated nested PCR products was carried out using the QIAquick gel extraction kit protocol following the manufacturer's instructions (Qiagen). The purified products were sequenced employing Applied Biosystems Big-Dye Ver
3.1 chemistry on an Applied Biosystems model 3730 automated capillary DNA sequencer.

Nuclear DNasel digests. To examine changes in chromatin structure of the inf1 locus, we performed nuclear DNaseI digestion studies. Mycelium was grown for 14 days in modified Plich medium (with or without $50 \mu \mathrm{M} 5$-AC and $5 \mu \mathrm{M}$ TSA) and was macerated while frozen in liquid $\mathrm{N}_{2}$. Exactly $2.0 \mathrm{~g}$ of the powder was transferred to a $50 \mathrm{ml}$ vial containing $10 \mathrm{ml}$ nuclease digestion buffer as described by van Blokland et al. (1997). Powder and buffer were mixed for $5 \mathrm{~min}$, after which a $400 \mu \mathrm{l}$ aliquot was retrieved (time point 0 ). To the remaining suspension, $4.0 \mu \mathrm{l}$ DNaseI ( 1 unit $\mu \mathrm{l}^{-1}$; Roche) was added and this was incubated at $37^{\circ} \mathrm{C}$. Aliquots of $400 \mu \mathrm{l}$ were taken every $10 \mathrm{~min}$ and added to the stop buffer during a $2 \mathrm{~h}$ period, and after 2.5, 3 and $4 \mathrm{~h}$. After retrieval, the aliquots were immediately mixed with $80 \mu \mathrm{l}$ stop buffer (van Blokland et al., 1997) and stored in liquid nitrogen. Genomic DNA was isolated using a phenol/chloroform extraction method (Raeder \& Broda, 1985) and dissolved in $100 \mu \mathrm{l}$ water. A PCR reaction was performed with $1 \mu \mathrm{l}$ DNA and primers that can amplify the endogenous inf1 gene sequence (primer SJS1, 5'-TTACAGTCGCGACGATGTCG-3'; SJS2, $5^{\prime}$-TCATAGCGACGCACACGTAG-3'). This amplification results in a product of $533 \mathrm{bp}$ from the endogenous inf1 gene only if DNaseI does not digest the corresponding sequence. Controls included PCR with primers SJS3 (5'-TTGGCAACAGATCTCCAAGC-3') and SJS4 (5'-TTCCTTACCGATGAGCGAGG-3') which amplify an $864 \mathrm{bp}$ product of the actin gene (Unkles et al., 1991). Experiments were performed in triplicate.

Southern and Northern analysis. Genomic DNA of $P$. infestans was isolated from mycelium as described by Raeder \& Broda (1985) with minor modifications. DNA samples $(6 \mu \mathrm{g})$ were digested for at least $5 \mathrm{~h}$ with 60 units of restriction enzyme (NEB), fractionated on $1 \%$ agarose gels, transferred to nylon membranes and probed as described previously (van West et al., 1998). For small DNA fragment hybridizations (methylation-sensitive restriction enzyme assays), $2.3 \mathrm{~kb}$ BamHI-digested genomic DNA containing the endogenous inf1 gene was isolated as described above. Subsequently, the genomic fragments were digested with 10 units of methylation-sensitive restriction enzymes, fractionated on $10 \%$ polyacrylamide/TBE gels (Bio-Rad), transferred to nylon membranes by means of an electroblotting system (Owl Separating Systems), and hybridized to the inf1 probes. DNA templates for probe synthesis were a $354 \mathrm{bp}$ NcoI-KpnI fragment of pHIN26 (van West et al., 1999) containing the inf1 coding sequence, $1395 \mathrm{bp}$ of pVW6-9 containing the promoter and coding regions of the inf1 gene, and a 796 bp HindIII fragment from pSTA31 (Unkles et al., 1991) containing the actin (actA) coding sequence. Probes were radiolabelled with $\left[\alpha_{-}{ }^{32} \mathrm{P}\right] \mathrm{dCTP}$ by using the Random Primers DNA labelling system (Gibco-BRL). For Northern blotting, total RNA from $P$. infestans was isolated, blotted and hybridized as described previously (van West et al., 1998).

\section{RESULTS}

\section{Methylation of the endogenous inf1 gene in silenced strains}

Modification of DNA and changes in chromatin structure provide direct and powerful mechanisms to regulate gene expression and can influence the transcriptional machinery by bringing about transcriptional repression. Cytosine methylation of DNA is one of many modifications influencing the transcriptional activity of a gene. To investigate whether the inf1 locus exhibits DNA cytosine 
methylation, we carried out a methylation-sensitive restriction analysis of the inf1 gene (GenBank accession no. AY830090). Previously, the coding sequence in the inf1-silenced strains was analysed using methylationsensitive restriction enzymes, but no cytosine or adenosine methylation could be detected, neither in the endogenous gene nor in the transgenes (van West et al., 1999). Genomic DNA isolated from the wild-type (88069) and four silenced strains (PY31, PY37, SY21 and H1) was digested with several methylation-sensitive restriction enzymes and analysed by Southern analysis using the inf1 promoter and coding regions as probes. The following restriction enzymes were used: $A l u \mathrm{I}, B s t \mathrm{UI}, F n u 4 \mathrm{HI}, M n l \mathrm{I}, M s p \mathrm{I}$, Sau3A and Sau96AI, representing 28 potential methylation sites in the promoter sequence. By comparing the hybridization patterns of 88069 with the inf1-silenced strains, no shifts in hybridizing bands were noted, suggesting absence of methylation at all tested sites (Fig. 1 and data not shown).

To investigate further whether cytosine methylation is involved in gene silencing in $P$. infestans, we performed expression studies with inf1-silenced strains grown in the presence of 5-AC (Fig. 2). This cytosine analogue can inhibit methyltransferases when incorporated into DNA (Kass et al., 1997). P. infestans isolates 88069 (wild-type strain), a control transformant (Y15), two transgenic inf1silenced transformants (PY31 and SY21), and one nontransgenic inf1-silenced transformant (H1) were examined (van West et al., 1999). 5-AC was added to the growth medium at different concentrations (up to $200 \mu \mathrm{M}$ ) and cultures were allowed to grow for 21 days. Considerable reduction in colony radial growth of all $P$. infestans isolates was observed at concentrations of 5-AC higher than $50 \mu \mathrm{M}$, suggesting toxicity of the drug towards $P$. infestans. However, little growth reduction was noted at concentrations lower than $50 \mu \mathrm{M}$ (data not shown), and therefore we used $50 \mu \mathrm{M} 5$-AC to determine its effect on inf1 gene expression in the silenced strains. Expression was analysed by Northern analysis and relative mRNA levels were quantified by phosphoimaging. The amount of inf1 mRNA in the wild-type strain grown in the absence of 5AC was set at $100 \%$ and the level of actin mRNA expression in each sample was used to correct for differences in loading.

Growth in the presence of $50 \mu \mathrm{M} 5$-AC resulted in a significant increase of inf1 expression in several of the silenced and non-silenced strains as determined by Northern analyses with phosphoimaging (Fig. 2). inf1 mRNA levels in the wild-type strain grown in the presence of 5-AC increased 2.3-fold compared to the control level. The antisense transformant PY31 showed an expression level of $20 \%$, compared to $0.5 \%$ in untreated mycelium, representing a 40 -fold increase after treatment with 5-AC. The non-transgenic, inf1-silenced strain $\mathrm{H} 1$ exhibited an 80 -fold increase, compared to the untreated mycelium. The relative amounts of inf1 mRNA were low, upon treatment with 5-AC in SY21 (0.5\%), when compared to the

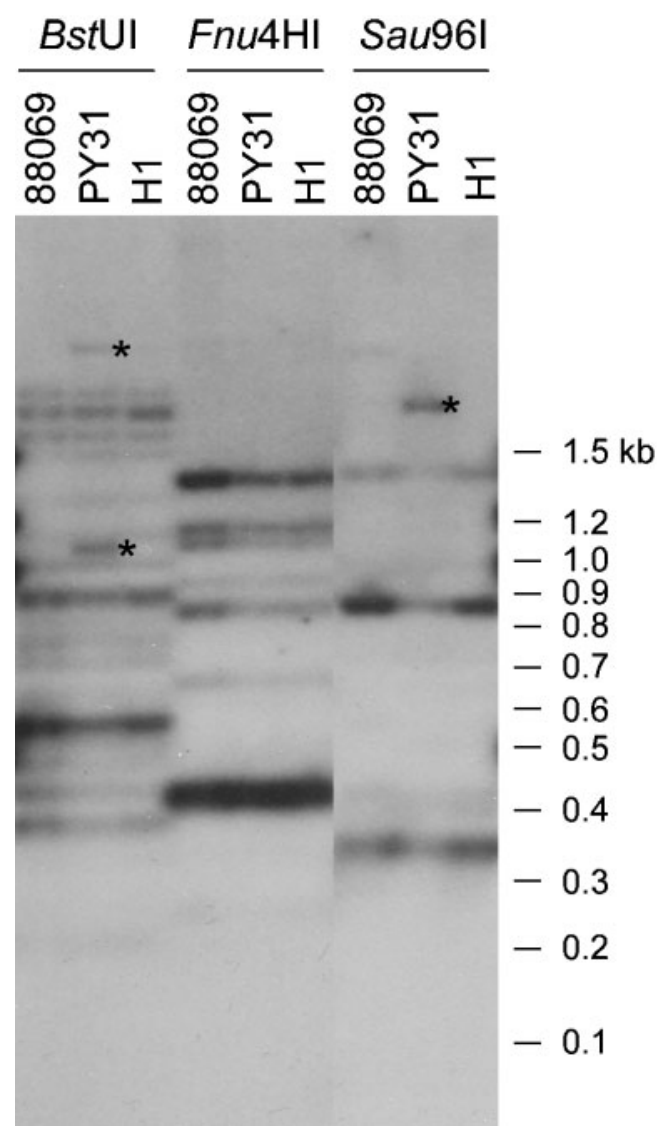

Fig. 1. Can cytosine methylation at the inf1 locus be detected? Methylation-sensitive restriction analysis by TBE-agarose gel electrophoresis and Southern blotting of genomic DNA isolated from the wild-type strain 88069 (wt), a transgenic inf1-silenced strain (PY31) and a non-transgenic inf1-silenced strain $(\mathrm{H} 1)$. Genomic DNA was digested with BstUI, Fnu4HI and Sau96I and than separated on a gel. A $1395 \mathrm{bp}$ fragment containing the promoter and coding regions of the inf1 gene was used as probe. Unique transgenic inf1 DNA fragments from the coding region [derived from the transformation vector pHIN28 (van West et al., 1999)] are indicated with an asterisk $\left(^{*}\right)$, the other hybridizing bands represent products derived from both the endogenous and transgenic inf1 gene sequences.

wild-type strain. However, compared to the untreated mycelium of SY21, expression in this transformant increased six times.

Following these results we decided to perform bisulfite treatment of genomic DNA and sequence a large section of the endogenous inf1 locus to detect any potential methylated cytosines. We sequenced an area of the promoter of up to $265 \mathrm{bp}$ upstream from the ATG start codon and a further $300 \mathrm{bp}$ downstream in the coding region from bisulfite-treated genomic DNA from the wildtype strains 88069 and Y15, and the inf1-silenced strains PY31, PY37 and H1. Remarkably, we were not able to 

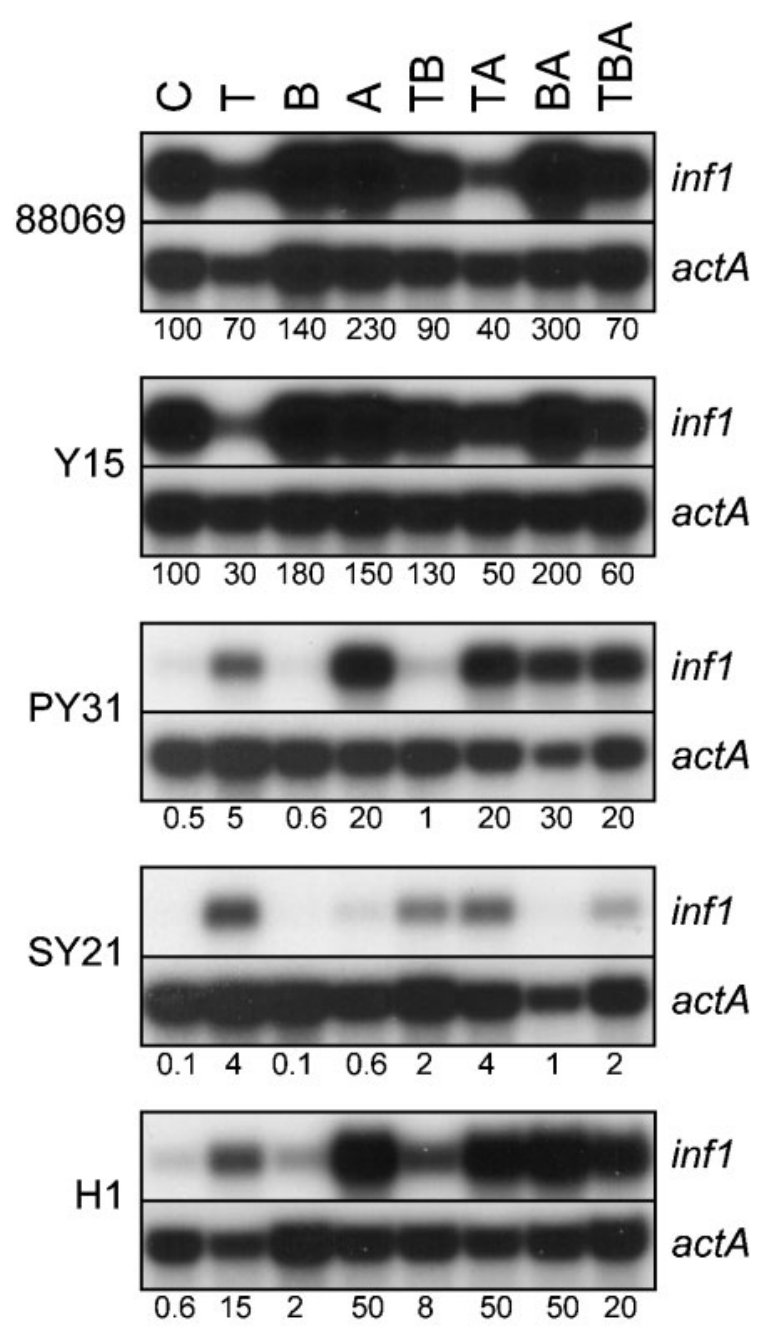

Fig. 2. Reversion of inf1 gene silencing in silenced strains of $P$. infestans. Analysis of inf1 mRNA production in wild-type strain (88069), in a transgenic antisense strain (PY31), in a cDNA transformant (SY21), in a non-transgenic silenced strain $(\mathrm{H} 1)$ and in a G418-resistant non-silenced strain (Y15), after addition of a DNA methylation inhibitor [50 $\mu \mathrm{M} 5-\mathrm{AC},(\mathrm{A})]$ or HDAC inhibitors [5 mM BuA (B), or $5 \mu \mathrm{M}$ TSA (T)], or combinations of two of these chemicals (TB, TA and BA), or all three (TBA). Northern blots, containing $15 \mu \mathrm{g}$ total RNA isolated from mycelium grown in vitro for 21 days, were hybridized with inf1 and actin (actA) probes. Relative amounts of inf1 mRNA corrected for loading errors (using the actin hybridizations) and compared to the level of inf1 mRNA of untreated wild-type $P$. infestans $(100 \%)$ are given below the autoradiographs.

detect any methylated cytosine residues in all tested DNA samples (data not shown).

These data indicate that, although 5-AC reverses inf1 silencing, methylation of cytosines does not take place in the inf1 locus.

\section{Relief of inf1 silencing with histone deacetylase (HDAC) inhibitors}

In general, acetylated regions of chromatin are transcriptionally active, whereas regions that are hypoacetylated are inactive (Katan-Khaykovich \& Struhl, 2002). Enzymes such as HDACs are believed to repress transcription by removing the acetyl groups from the $\mathrm{N}$-terminal tails of the core histones of the chromatin. Several reports demonstrate that inhibition of HDACs by drugs, such as trichostatin-A (TSA) and $\mathrm{BuA}$, results in reactivation of transcriptionally silenced genes (Selker, 1998; Ayer, 1999).

We used HDAC inhibitors to determine whether reactivation of the inf1-silenced state could be achieved in $P$. infestans. Therefore, we analysed expression of inf1 in several inf1-silenced strains grown in the presence of TSA $(3.3 \mu \mathrm{M})$ and $\mathrm{BuA}(5 \mathrm{mM})$ (Fig. 2). Similar to the experiments described above for 5 -AC, we first determined the highest concentration of the drugs that had little or no toxic effects by colony radial growth assays (data not shown). Addition of BuA resulted in some increase of expression of the inf1 gene in the wild-type and control (Y15) strains (1.4- and 1.8-fold, respectively). Similarly, little or no increases in expression levels were observed in the inf1-silenced strains SY21, PY31 and H1 after treatment with BuA.

Addition of TSA to the culture medium resulted in reduced inf1 expression in the wild-type and control strains, whereas an increase of infl expression was noted in all silenced strains. For example, in the inf1 cDNA transformant (SY21), expression increased more than 40-fold, and a 25 -fold increase was noted in the non-transgenic inf1silenced strain (H1) (Fig. 2).

To investigate whether combinations of the drugs work synergistically in reactivating inf1 expression, we performed expression studies with combinations of 5-AC, TSA and $\mathrm{BuA}$. Analysing the results of these experiments did not reveal any obvious synergistic activities as none of the combined treatments resulted in an increased inf1 expression level when compared to the highest level that was measured for the mycelial samples that were treated with only one inhibitor compound. The only exception is in mycelium of PY31 when this was treated with both BuA and TSA. However, in this case, only a $50 \%$ increase was observed when compared to PY31 mycelium treated with TSA alone.

We also investigated whether exposure to other chemicals involved in DNA synthesis and degradation had any effect on reactivating inf1 gene expression. The DNA synthesis inhibitor hydroxy-urea was tested at concentrations of up to $50 \mathrm{mM}$, but no reactivation of inf1 gene expression was found. Similarly, concentrations of up to $100 \mu \mathrm{M}$ of the nuclease inhibitor aurintricarboxylic acid (ATA) did not result in reactivation (data not shown).

From these experiments we can conclude that growth of the inf1-silenced strains in the presence of TSA leads to 
reversion of the inf1-silenced state, suggesting that the silenced state is, at least partly, the result of hypoacetylated histones.

\section{Changes in chromatin structure}

To investigate whether silencing had led to changes in the chromatin structure at the endogenous inf1-silenced locus, we performed DNaseI digestion experiments of isolated nuclei. It has been demonstrated that densely packed heterochromatin is less accessible to nucleases (Grewal \& Elgin, 2002). Nuclear digests were performed with three inf1-silenced strains, PY31, PY37 and H1, and wild-type strain 88069 as a control. A time-course experiment was performed by taking samples from the DNaseI-treated suspensions every $10 \mathrm{~min}$ over a $2 \mathrm{~h}$ period, and after 2.5, 3 and $4 \mathrm{~h}$. Subsequently, the genomic DNA was isolated and PCR was performed to test for the presence of the intact inf1 gene sequence (Fig. 3a). We used a primer combination that only amplified the complete endogenous infl gene sequence. Transgenic sequences could not be amplified. As a control, we also used primers to amplify the actin gene. After $30 \mathrm{~min}$, the control actin gene could not be amplified in DNaseI-treated DNA that was isolated from the wildtype and all inf1-silenced strains. infl could not be amplified in the wild-type strain after $30 \mathrm{~min}$. However, inf1 could be amplified in all tested inf1-silenced strains up to $90 \mathrm{~min}$ after DNaseI treatment. These findings indicate that the chromatin present in the endogenous infl locus is more densely packed in the infl-silenced strains compared to the wild-type and control strains.

We then decided to investigate whether nuclear DNaseI treatment of isolated nuclei from inf1-silenced lines isolated from mycelium grown in the presence of TSA and 5-AC would result in increased digestion efficiency. We found for PY31, PY37 and 88069 (Fig. 3b and data not shown) that the actin gene could be amplified up to 20 min after DNaseI treatment, which is identical to non-treated nuclei of these strains (Fig. 3a). However, the inf1 gene could only be amplified up to $20 \mathrm{~min}$ after DNaseI treatment for all three tested strains (Fig. 3b and data not shown), whereas in the non-treated experiment (Fig. 3a), the inf1 gene could be amplified up to 90 min following DNaseI treatment. This provides further evidence that the infl locus is indeed densely packed in the inf1-silenced lines and that adding TSA and 5-AC to the growth medium allows the chromatin to remodel back to a less condensed state.

\section{Reactivated transgenic strains revert back to the silenced state, while non-transgenic strains do not}

To investigate whether reactivated inf1 expression was maintained in the silenced strains after treatment with 5AC and TSA, we performed experiments in which we grew transgenic inf1-silenced strains (PY37 and SY21) and a

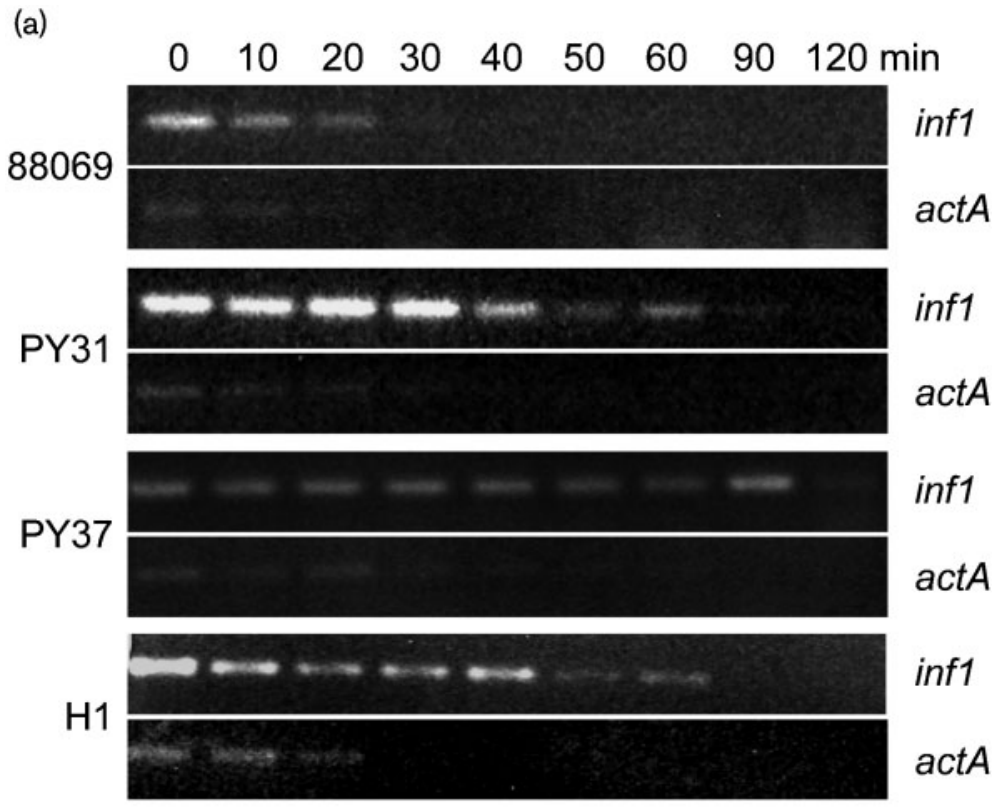

(b)

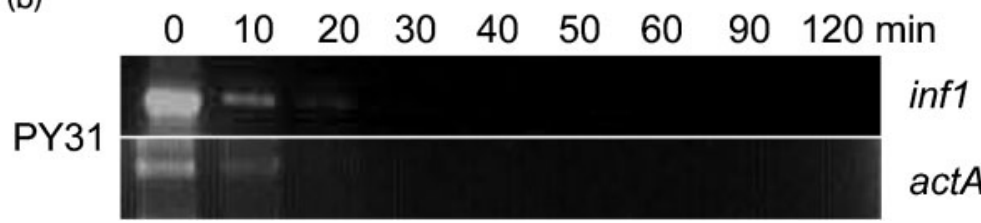

Fig. 3. Genomic DNA at the endogenous inf1 locus of inf1-silenced strains is less accessible to DNasel digestion. Genomic DNA of inf1silenced lines was isolated from identical amounts of nuclei that were treated with DNasel over a time-course of $120 \mathrm{~min}$. The nuclei were isolated from mycelium that had been grown in Plich medium without (a) and with $50 \mu \mathrm{M} 5-\mathrm{AC}$ and $5 \mu \mathrm{M}$ TSA (b). PCR was performed on the isolated DNA with inf1- and actin-specific primers and the amplified products were run on a $1 \%$ agarose gel. The presence of the amplified product indicates that DNasel had not restricted the inf1 or actin gene sequences. The inf1-silenced strains show a delay in DNasel digestion of the endogenous inf1 gene compared to the nonsilenced wild-type (a), whereas the 5-AC- and TSA-treated strain PY31 (b) does not show a delay in DNasel digestion, suggesting that treatment with these compounds resulted in less condensed DNA in the inf1 locus. 
non-transgenic inf1-silenced strain (H1) in the presence of 5-AC and TSA on solidified RS-medium for 21 days. Subsequently, pieces of mycelia were transferred to fresh liquid medium with and without the drugs for a further period of growth for 21 days. Total RNA was isolated and inf1 expression was analysed by Northern blotting (Fig. 4a). Untreated mycelium was used as a control. The inf1 transgenic strains PY37 and SY21 showed an increase of inf1 expression during growth in medium containing 5-AC and TSA, whereas inf1 expression decreased again after transfer to medium without the drugs. Interestingly, the inf1 non-transgenic silenced strain (H1) also resumed inf1 expression upon growth in medium containing the drugs, but expression of the inf 1 gene continued after transfer to fresh medium in the absence of these drugs. Furthermore, we noticed that the non-transgenic, inf1-silenced strains gradually reverted to the wild-type state of INF1 protein production during subculturing over a period of 18 months. We investigated this in more detail by performing Northern analysis of several of these strains which had been subcultured and grown for 18 months, and compared these with the same isolates that had been stored in liquid $\mathrm{N}_{2}$ soon after they had been generated (Fig. 4b). All tested strains showed a higher inf1 expression level when compared to the same isolate stored in liquid $\mathrm{N}_{2}$, indicating a loss of the silenced state over time.

These findings demonstrate that the presence of inf1 transgenic sequences is required to maintain the inf1silenced state.

\section{DISCUSSION}

Here we describe evidence for chromatin remodelling and histone deacetylation controlling gene expression in the oomycete $P$. infestans. Our findings suggest that silencing of the inf1 gene is accomplished by a hypoacetylated state of histones at the inf1 locus, resulting in the formation of heterochromatin. Cytosine methylation does not seem to occur, although the cytosine methylation inhibitor 5azacytidine is able to reverse the silenced state temporarily.

DNA methylation is important for regulating genome functions and differential control of gene expression in eukaryotes such as plants, fungi and mammals. In total we have tested almost 50 methylation-sensitive restriction sites within the inf1 gene without detection of any methylated cytosines (van West et al., 1999; this study). Furthermore, methylation-sensitive sequencing of the infl locus did not reveal any methylated cytosine in the inf1-silenced strains. These results are in line with observations made by Judelson \& Whittaker (1995), who found that transgenes in $P$. infestans became inactivated without noticeable methylation of the transgenic DNA sequences.

Interestingly, for a long time, several organisms including yeast, C. elegans and Drosophila have been reported to lack detectable methylation of their genomes (Lyko, 2001). For example, C. elegans does not seem to have a conventional DNA methyltransferase-like gene (Bird, 2002). However, it was discovered that at certain stages in the developmental process of Drosophila, methylated cytosine bases are (a)

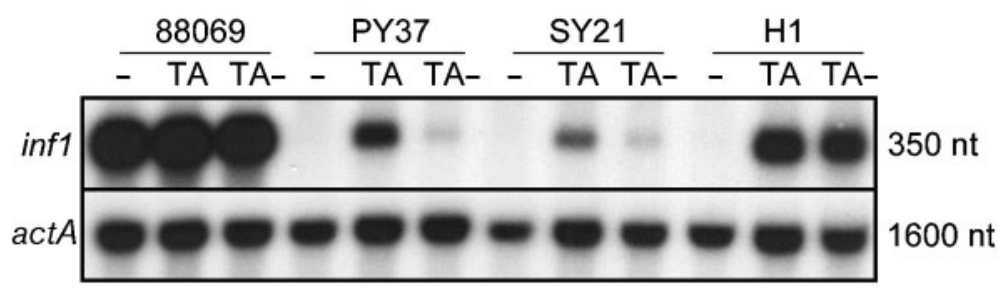

(b)

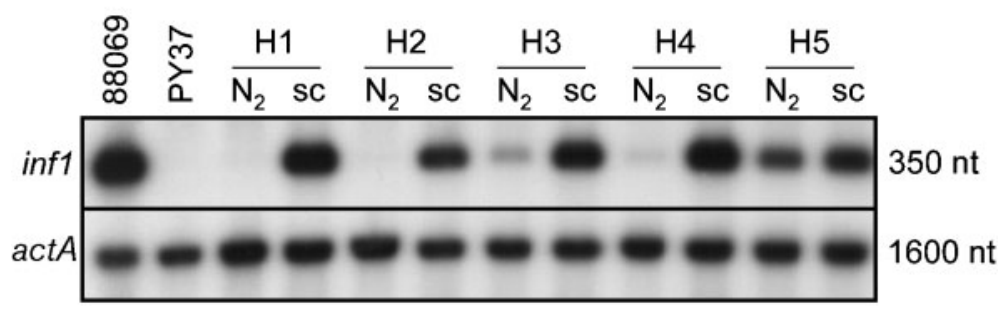

Fig. 4. Stable reversion of inf1 gene silencing only occurs in non-transgenic inf1-silenced strains after treatment with TSA and 5-AC, or over time. (a) Analysis of inf1 mRNA production in mycelium of wild-type strain (88069), in transgenic antisense strains (PY37), in a cDNA transformant (SY21) and in a nontransgenic silenced strain $(\mathrm{H} 1)$ before treatment (-), after addition of $50 \mu \mathrm{M} 5-\mathrm{AC}$ and $5 \mu \mathrm{M}$ TSA) (TA) and after transfer again to medium without 5-AC and TSA (TA-). Only the non-transgenic silenced line $\mathrm{H} 1$ reverses to wild-type levels of inf1 expression after treatment with 5-AC and TSA. (b) Analysis of inf1 mRNA production in mycelium of wildtype strain (88069), in transgenic antisense strains (PY37) and in five individual nontransgenic silenced strains $(\mathrm{H} 1-5)$ after storage in liquid nitrogen $\left(\mathrm{N}_{2}\right)$ and after subculturing for 18 months (sc). Northern blots, containing $15 \mu \mathrm{g}$ total RNA, were hybridized with inf1 and actin (actA) probes. Transcript lengths are indicated in nucleotides (nt). All non-transgenic silenced lines revert to wildtype levels of inf1 expression after prolonged subculturing. 
present. Apparently, methylation occurs in Drosophila at unusual target sequences not including traditional $\mathrm{CpG}$ sites (Lyko et al., 2000). At present we have not been able to identify a good DNA methyltransferase candidate gene in the available genome sequences of $P$. infestans, based on BLAST searches (www.broad.mit.edu/annotation/genome/ phytophthora_infestans/Home.html).

Interestingly, addition of 5-AC to the growth medium resulted in reactivation of inf1 gene expression in silenced strains. The fact that we were unable to demonstrate any DNA methylation using restriction analysis or bisulfite sequencing suggests that the inhibitor must function in a different way to release silencing. Perhaps one explanation could be that the incorporation of the 5-AC residue in the inf1 locus enables the generation of a more open conformation of the inf1 gene, which results in an increase of infl expression. Alternatively, there is good evidence that 5-AC can also inhibit histone methylation (Wada et al., 2005; Wozniak et al., 2006). Therefore, adding 5-azacytidine into the culture medium may have had a strong effect in reducing methylation of the histones in the inf1 locus, resulting in the release of silencing.

Acetylation, deacetylation, ubiquitination, phosphorylation and methylation of histones have all been implicated in activation and silencing of transcription (Grewal \& Elgin, 2002; Fischle et al., 2003). The different histone modifications and the corresponding enzymic systems have been studied extensively, and this has resulted in a complex picture of how eukaryotic cells establish and maintain local and global patterns of chromatin modification. It is generally accepted that hyperacetylated histones are associated with activated genome regions, whereas deacetylation of histones leads to repression and silencing (Fischle et al., 2003).

Here we set out to investigate whether we are able to reverse inf1 gene silencing by inactivating HDACs. In many eukaryotic systems, long-term treatment with BuA, TSA or other HDAC inhibitors can lead to complete shifts from predominantly non-acetylated to largely multi-acetylated histones (Waterborg, 1998 and references therein). Addition of TSA to the growth medium of inf1-silenced strains resulted in reactivation of inf1 gene expression in the tested strains (Fig. 1), demonstrating that inf1 silencing in $P$. infestans is correlated with a hypoacetylated state of histone molecules.

Interestingly, addition of BuA alone did not result in an increase of inf1 gene expression in the transgenic inf1silenced strains. Only very little reactivation could be observed in the non-transgenic inf1-silenced strain, possibly indicating that $\mathrm{BuA}$ is a poor inhibitor of HDAC in $P$. infestans. Similar results have been found in Chlamydomonas reinhardtii (Waterborg, 1998). In this alga, TSA was also able to induce hyperacetylation of histones, whereas BuA was ineffective (Waterborg, 1998). Combinations of two or three inhibitors added to the growth medium resulted in no clear synergistic reactivation of infl expression.
To determine whether reactivation of infl expression is stable after treatment with TSA and 5-AC, we subcultured the treated strains on media without TSA and 5-AC. This resulted in restoration of silencing in all strains with the exception of the non-transgenic silenced strains. Therefore, the presence of transgenic inf1 sequences was essential to re-establish the silenced state. Furthermore we noticed that silencing was lost in the non-transgenic inf1-silenced strains over prolonged subculturing.

These findings demonstrate that the acetylation status of histones is inherited during vegetative growth, but that it can be modulated by external or internal factors. It seems that the cell has a regulatory system that allows it to monitor the acetylation status of its histones. Experiments in yeast have suggested the presence of a system that maintains a dynamic balance of acetylation of histone proteins (Vogelauer et al., 2000; Katan-Khaykovich \& Struhl, 2002). It was found that acetylation and deacetylation reactions occur continuously, thereby generating a steady-state level of global or bulk histone acetylation. This homeostasis can be disturbed locally by the recruitment of histone acetylases or HDACs to DNA-binding proteins linked to the chromatin (Struhl, 1998). It is thought that once recruitment and activity is finished, the cell rapidly restores its initial acetylation status by allowing globally acting enzymes to restore the acetylation equilibrium (Katan-Khaykovich \& Struhl, 2002).

Modification of gene expression through alteration of chromatin structure has been found in many eukaryotic organisms, including nematodes (Ryner \& Swain, 1995; Kelly \& Fire, 1998), Drosophila (Weissmann \& Lyko, 2003), plants (van Blokland et al., 1997), mammals (Bird \& Wolffe, 1999), yeasts and fungi (Roth, 1995; Selker, 1998). A common method to demonstrate a more condensed state of chromatin at a particular locus is to treat intact nuclei with nucleases in a time-course experiment and to assess the rate of DNA degradation. If the DNA is less accessible for nucleases, a delay in DNA degradation is observed (Grewal \& Elgin, 2002). We observed such a delay in DNA degradation in both the transgenic and non-transgenic inf1-silenced strains compared to non-silenced strains. These results demonstrate that inf1 silencing is associated with a condensed chromatin state of the infl locus.

We demonstrated previously that the infl-silenced state could be transmitted in heterokaryotic strains from transgenic inf1-silenced nuclei to wild-type nuclei (van West et al., 1999). Furthermore, using run-on assays we demonstrated that inf1 silencing is regulated at the transcriptional level and therefore we could not explain the observed internuclear gene-silencing phenomenon as a post-transcriptional silencing process.

Whisson et al. (2005) demonstrated that efficient silencing is obtained in P. infestans when protoplasts are treated with dsRNA of a target gene. Typically, about 15 days posttreatment silencing appears to reach maximum levels and normal gene expression levels resume gradually thereafter. 
At present it is unclear whether this silencing process is based upon mRNA degradation or whether transcriptional silencing processes play a role. Indeed, several studies indicate that eukaryotes may require dsRNA and siRNA (small interfering RNA) to induce TGS (Cerutti, 2003; Vermaak et al., 2003; Morris et al., 2004). Evidence from fission yeast shows that repetitive sequences found at the centromeres produce dsRNAs that are involved in the formation and maintenance of heterochromatin (Vermaak et al., 2003; Volpe et al., 2002; Reinhart \& Bartel, 2002).

We hypothesize that integration of homologous transgenes in the genome of $P$. infestans results in the formation of aberrant RNA molecules (van West et al., 1999). These aberrant RNA molecules may be dsRNA molecules, or they may be used as a template to generate new dsRNA via an RNA-dependent RNA polymerase (Dalmay et al., 2000; Beclin et al., 2002). The dsRNA molecules may be cleaved by a homologue of Dicer into siRNAs. Database searches against genome sequences from $P$. infestans (http:// www.broad.mit.edu/annotation/genome/phytophthora infestans/Home.html) do suggest that Dicer and RNAdependent RNA polymerase candidate genes may be present. It is then speculated that the generated siRNAs induce changes in the chromatin structure of homologous sequences, which has already been described in several systems (Nykänen et al., 2001; Mette et al., 2000; Dalmay et al., 2000; Vaistij et al., 2002; Brodersen \& Voinnet, 2006). It is thought that the siRNA may act as a guide to activate enzymes involved in modulating the methylation status of histones and/or to activate HDACs, which would remove the acetyl groups on the homologous DNA sequence (Pickford \& Cogoni, 2003). Once the siRNAs are formed in the cell, they may be imported into other nuclei in heterokaryotic cells, where, subsequently, a similar condensed chromatic silenced state is induced. We are currently testing this model.

\section{ACKNOWLEDGEMENTS}

This work was financially supported by the BBSRC [grants 1/P07720 (P.v. W., S. J.S. and N.A.R. G.), BRE13669, (A. A. A., N.A.R. G. and P.v.W.), 1/P17160 (L. J.G.-B. and P.v.W.) and BB/C513977/1 (P.v.W.), and a studentship to C.A.W.], Syngenta (studentship to S. L.), and The Royal Society (personal fellowship to P.v.W.). We would also like to thank John van't Klooster and Sophien Kamoun for assistance in the initial phases of this research, and Peter van der Voort for help with culturing $P$. infestans strains.

\section{REFERENCES}

Ayer, D. E. (1999). Histone deacetylases: transcriptional repression with SINers and NuRDs. Trends Cell Biol 9, 193-198.

Baulcombe, D. (2002). RNA silencing. Curr Biol 12, R82-R84.

Beclin, C., Boutet, S., Waterhouse, P. \& Vaucheret, H. (2002). A branched pathway for transgene-induced RNA silencing in plants. Curr Biol 12, 684-688.

Bird, A. (2002). DNA methylation patterns and epigenetic memory. Genes Dev 16, 6-21.
Bird, A. P. \& Wolffe, A. P. (1999). Methylation-induced repression belts, braces, and chromatin. Cell 99, 451-454.

Brodersen, P. \& Voinnet, O. (2006). The diversity of RNA silencing pathways in plants. Trends Genet 22, 268-280.

Cerutti, H. (2003). RNA interference: traveling in the cell and gaining functions? Trends Genet 19, 39-46.

Chandler, V. L., Eggleston, W. B. \& Dorweiler, J. E. (2000). Paramutation in maize. Plant Mol Biol 43, 121-145.

Cogoni, C. \& Macino, G. (1999). Homology-dependent gene silencing in plants and fungi: a number of variations on the same theme. Curr Opin Microbiol 2, 657-662.

Cogoni, C., Irelan, J. T., Schumacher, M., Schmidhauser, T. J., Selker, E. U. \& Macino, G. (1996). Transgene silencing of the al-1 gene in vegetative cells of Neurospora is mediated by a cytoplasmic effector and does not depend on DNA-DNA interactions or DNA. EMBO J 15, 3153-3163.

Dalmay, T., Hamilton, A., Rudd, S., Angell, S. \& Baulcombe, D. C. (2000). An RNA-dependent RNA polymerase gene in Arabidopsis is required for posttranscriptional gene silencing mediated by a transgene but not by a virus. Cell 101, 543-553.

Fire, A., Xu, S.-Q., Montgomery, M. K., Kostas, S. A., Driver, S. E. \& Mello, C. C. (1998). Potent and specific genetic interference by doublestranded RNA in Caenorhabditis elegans. Nature 391, 806-811.

Fischle, W., Wang, Y. \& Allis, C. D. (2003). Histone and chromatin cross-talk. Curr Opin Cell Biol 15, 172-183.

Grewal, S. I. S. \& Elgin, S. C. R. (2002). Heterochromatin: new possibilities for the inheritance of structure. Curr Opin Genet Dev 12, 178-187.

Henikoff, S. (1998). Conspiracy of silence among repeated transgenes. Bioessays 20, 532-535.

Hollick, J. B., Dorweiler, J. E. \& Chandler, V. L. (1997). Paramutation and related allelic interactions. Trends Genet 13, 302-308.

Judelson, H. S. \& Whittaker, L. S. (1995). Inactivation of transgenes in Phytophthora infestans is not associated with their deletion, methylation, or mutation. Curr Genet 28, 571-579.

Kass, S. U., Pruss, D. \& Wolffe, A. P. (1997). How does DNA methylation repress transcription? Trends Genet 13, 444-449.

Katan-Khaykovich, Y. \& Struhl, K. (2002). Dynamics of global histone acetylation and deacetylation in vivo: rapid restoration of normal histone acetylation status upon removal of activators and repressors. Genes Dev 16, 743-752.

Kelly, W. G. \& Fire, A. (1998). Chromatin silencing and the maintenance of a functional germline in Caenorhabditis elegans. Development 125, 2451-2456.

Kooter, J. M., Matzke, M. A. \& Meyer, P. (1999). Listening to the silent genes: transgene silencing, gene regulation and pathogen control. Trends Plant Sci 4, 340-347.

Lu, R., Martin-Hernandez, A. M., Peart, J. R., Malcuit, I. \& Baulcombe, D. C. (2003). Virus-induced gene silencing in plants. Methods 30, 296-303.

Lyko, F. (2001). DNA methylation learns to fly. Trends Genet 17, 169-172.

Lyko, F., Ramsahoye, B. H. \& Jaenisch, R. (2000). DNA methylation in Drosophila melanogaster. Nature 408, 538-540.

Mette, M. F., Aufsatz, W., van der Winden, J., Matzke, M. A. \& Matzke, A. J. (2000). Transcriptional silencing and promoter methylation triggered by double-stranded RNA. EMBO J 19, 5194-5201.

Morris, K. V., Chan, S. W., Jacobsen, S. E. \& Looney, D. J. (2004). Small interfering RNA-induced transcriptional gene silencing in human cells. Science 305, 1289-1292. 
Nakayashiki, H. (2005). RNA silencing in fungi: mechanisms and applications. FEBS Lett 579, 5950-5957.

Nykänen, A., Haley, B. \& Zamore, P. D. (2001). ATP requirements and small interfering RNA structure in the RNA interference pathway. Cell 107, 309-321.

Pickford, A. S. \& Cogoni, C. (2003). RNA-mediated gene silencing. Cell Mol Life Sci 60, 871-882.

Plasterk, R. H. \& Ketting, R. F. (2000). The silence of the genes. Curr Opin Genet Dev 10, 562-567.

Raeder, U. \& Broda, P. (1985). Rapid preparation of DNA from filamentous fungi. Lett Appl Microbiol 1, 17-20.

Reinhart, B. J. \& Bartel, D. P. (2002). Small RNAs correspond to centromere heterochromatic repeats. Science 297, 1831.

Roth, S. Y. (1995). Chromatin-mediated transcriptional repression in yeast. Curr Opin Genet Dev 5, 168-173.

Ruiz, M. T., Voinnet, O. \& Baulcombe, D. C. (1998). Initiation and maintenance of virus-induced gene silencing. Plant Cell 10, 937-946.

Ryner, L. C. \& Swain, A. (1995). Sex in the '90s. Cell 81, 483-493.

Selker, E. U. (1997). Epigenetic phenomena in filamentous fungi: useful paradigms or repeat-induced confusion. Trends Genet 13, 296-301.

Selker, E. U. (1998). Trichostatin A causes selective loss of DNA methylation in Neurospora. Proc Natl Acad Sci U S A 95, 9430-9435.

Struhl, K. (1998). Histone acetylation and transcriptional regulatory mechanisms. Genes Dev 12, 599-606.

Tyler, J. K. \& Kadonaga, J. T. (1999). The 'dark side' of chromatin remodelling: repressive effects on transcription. Cell 99, 443-446.

Unkles, S. E., Moon, R. P., Hawkins, A. R., Duncan, J. M. \& Kinghorn, J. R. (1991). Actin in the oomycetous fungus Phytophthora infestans is the product of several genes. Gene 100, 105-112.

Vaistij, F. E., Jones, L. \& Baulcombe, D. C. (2002). Spreading of RNA targeting and DNA methylation in RNA silencing requires transcription of the target gene and a putative RNA-dependent RNA polymerase. Plant Cell 14, 857-867.

van Blokland, R., ten Lohuis, M. \& Meyer, P. (1997). Condensation of chromatin in transcriptional regions of an inactivated plant transgene: evidence for an active role of transcription in gene silencing. Mol Gen Genet 257, 1-13. van der Krol, A. R., Mur, L. A., Beld, M., Mol, J. N. \& Stuitje, A. R. (1990). Flavonoid genes in petunia: addition of a limited number of gene copies may lead to a suppression of gene expression. Plant Cell 2, 291-299.

van West, P., de Jong, A. J., Judelson, H. S., Emons, A. M. C. \& Govers, F. (1998). The ipiO gene of Phytophthora infestans is highly expressed in invading hyphae during infection. Fungal Genet Biol 23, $126-138$.

van West, P., Kamoun, S., van't Klooster, J. W. \& Govers, F. (1999). Internuclear gene silencing in Phytophthora infestans. Mol Cell 3, 339-348.

Vaucheret, H., Beclin, C., Elmayan, T., Feuerbach, F., Godon, C., Morel, J. B., Mourrain, P., Palauqui, J. C. \& Vernhettes, S. (1998). Transgene-induced gene silencing in plants. Plant J 16, 651-659.

Vermaak, D., Ahmad, K. \& Henikoff, S. (2003). Maintenance of chromatin states: an open-and-shut case. Curr Opin Cell Biol 15, 266-274.

Vogelauer, M., Wu, J., Suka, N. \& Grunstein, M. (2000). Global histone acetylation and deacetylation in yeast. Nature 408, 495-498.

Volpe, T. A., Kidner, C., Hall, I. M., Teng, G., Grewal, S. I. \& Martienssen, R. A. (2002). Regulation of heterochromatic silencing and histone H3 lysine-9 methylation by RNAi. Science 297, 1833-1837.

Wada, H., Kagoshima, M., Ito, K., Barnes, P. J. \& Adcock, I. M. (2005). 5-Azacytidine suppresses RNA polymerase II recruitment to the SLPI gene. Biochem Biophys Res Commun 331, 93-99.

Waterborg, J. H. (1998). Dynamics of histone acetylation in Chlamydomonas reinhardtii. J Biol Chem 273, 27602-27609.

Weissmann, F. \& Lyko, F. (2003). Cooperative interactions between epigenetic modifications and their function in the regulation of chromosome architecture. Bioessays 25, 792-797.

Whisson, S. C., Avrova, A. O., van West, P. \& Jones, J. T. (2005). A method for double-stranded RNA-mediated transient gene silencing in Phytophthora infestans. Mol Plant Pathol 6, 153-163.

Wozniak, R. J., Klimecki, W. T., Lau, S. S., Feinstein, Y. \& Futscher, B. W. (2006). 5-Aza-2-deoxycytidine-mediated reductions in G9A histone methyltransferase and histone H3 K9 di-methylation levels are linked to tumor suppressor gene reactivation. Oncogene 26, 77-90.

Edited by: I. K. Toth 